\title{
Evaluation of bone formation in neonatal mouse calvariae using micro-CT and histomorphometry: an in vitro study
}

DOI:

10.1016/j.acthis.2020.151614

\section{Document Version}

Accepted author manuscript

Link to publication record in Manchester Research Explorer

Citation for published version (APA):

Zheng, R., Song, J., Wu, X., \& Watts, D. C. (2020). Evaluation of bone formation in neonatal mouse calvariae using micro-CT and histomorphometry: an in vitro study. Acta Histochemica, 122(7), 151614.

https://doi.org/10.1016/j.acthis.2020.151614

\section{Published in:}

Acta Histochemica

\section{Citing this paper}

Please note that where the full-text provided on Manchester Research Explorer is the Author Accepted Manuscript or Proof version this may differ from the final Published version. If citing, it is advised that you check and use the publisher's definitive version.

\section{General rights}

Copyright and moral rights for the publications made accessible in the Research Explorer are retained by the authors and/or other copyright owners and it is a condition of accessing publications that users recognise and abide by the legal requirements associated with these rights.

\section{Takedown policy}

If you believe that this document breaches copyright please refer to the University of Manchester's Takedown Procedures [http://man.ac.uk/04Y6Bo] or contact uml.scholarlycommunications@manchester.ac.uk providing relevant details, so we can investigate your claim.

\section{OPEN ACCESS}




\section{Evaluation of bone formation in neonatal mouse calvariae using micro-CT and histomorphometry: an in vitro study}

Ren-jian Zheng ${ }^{\mathrm{a}, \mathrm{b}, \mathrm{c}}$, Jin-lin Song ${ }^{\mathrm{b}, \mathrm{c}, \mathrm{d}}$, Xiao-hong Wu $\mathrm{Wu}^{\mathrm{a}, \mathrm{b}, \mathrm{c}, *}$, David C. Watts $, \mathrm{e}, * * *$

${ }^{a}$ Department of Prosthodontics, Stomatological Hospital of Chongqing Medical University, No. 426 Songshibei Road, Yubei, Chongqing 401147, China ${ }^{\mathrm{b}}$ Chongqing Key Laboratory of Oral Diseases and Biomedical Sciences, Chongqing 401147, China

${ }^{\mathrm{c}}$ Chongqing Municipal Key Laboratory of Oral Biomedical Engineering of

Higher Education, Chongqing 401147, China ${ }^{\mathrm{d}}$ College of Stomatology,

Chongqing Medical University, Chongqing, China

${ }^{\mathrm{e}}$ School of Medical Sciences and Photon Science Institute, University of Manchester, Manchester M13 9PL, UK ${ }^{\mathrm{f}}$ Institute of Material Science and Technology, Friedrich-Schiller-University, Jena, Lobdergraben 32,

07743, Germany

* Corresponding author at: Department of Prosthodontics, Stomatological Hospital of Chongqing Medical University, No. 426 Songshibei Road, Yubei, Chongqing, 401147, China.

** Corresponding author at: School of Medical Sciences and Photon Science Institute,

University of Manchester, Manchester M13 9PL, UK.

E-mail addresses:

500222@hospital.cqmu.edu.cn (X.-h. Wu),

david.watts@manchester.ac.uk (D.C.Watts).

Published in Acta Histochemica 122 (2020) 151614

https://doi.org/10.1016/j.acthis.2020.151614

Published Versions

Accepted 10 August 2020

Available online 22 August 2020 


\begin{abstract}
Neonatal calvarial bone has been widely used for investigating the biological behaviour of intramembranous bones. This work evaluated the bone formation of neonatal calvarial bone by microcomputed tomography (micro-CT) and histomorphometry. Moreover, the viability of neonatal calvarial bone and the effect of micro-CT radiation exposure on neonatal calvarial bone viability were investigated. The calvarial bones of 4-day-old CD-1 mice were cultured in Dulbecco's modified Eagle's medium (DMEM) or osteogenic medium (OM) for 23 days. Micro-CT scanning and histological analysis were performed on days 2, 9, 16 and 23. An "OM-control" group was scanned only on days 2 and 23 to evaluate the effect of a single micro-CT radiation dose on calvarial bones. Histomorphometric measurements revealed that the number of osteoblasts per unit bone surface area (N. Ob/BS, $/ \mathrm{mm}^{2}$ ) (days 9, 16 and 23) and the number of osteoclasts per unit bone surface area (N. Oc/BS, $/ \mathrm{mm}^{2}$ ) (days 9 and 16) were higher and lower, respectively, in the OM group than in the DMEM group. Moreover, the calvarial bone survived for at least 16 days in vitro, as indicated by tartrate-resistant acid phosphatase (TRAP)-positive staining. Micro-CT assessment revealed that the bone surface (BS), bone volume (BV), bone surface density (BS/ TV(Tissue volume)) and percent bone volume (BV/TV) were greater in the OM group than in the DMEM group except at baseline on day 2. All bone parameters of calvariae cultured in OM and OM-control conditions were not significantly different on days 2 and 23. Thus, the radiation dose from micro-CT in our study design had no perceptible effect on the formation of mouse calvarial bone in vitro.
\end{abstract}

Keywords:

Neonatal mouse calvaria Bone formation Micro-CT

Histomorphometry 


\section{Introduction}

Neonatal mouse calvarial organ culture has been extensively used for investigating the biological behaviour of intramembranous bones. First, organ culture preserves three-dimensional architecture and cellular diversity to a great degree. Additionally, organ culture offers greater physiological relevance than in vitro cell line culture(Mohammad et al., 2008b). Mouse calvarial bone is, in general, easily obtained for culture, and the dissection and culture techniques are well-established procedures(Cochran and Rouse, 1993; Goldhaber and Rabadjija, 1991; Sakai et al., 2000). Calvarial bone is also advantageous in that it requires minimal preparation for optical microanalysis. The bone formation ability of mouse calvarial bone is dependent on the culture conditions, which influence cell proliferation and differentiation. Various culture media have been used to culture mouse calvarial bone, such as Dulbecco's modified Eagle's medium (DMEM), osteogenic medium (OM; DMEM with osteogenic supplements), Gwatkin and Judah (BGJb) medium, and modified BGJb medium(Garrett, 2003a; Liu et al., 2013; Wu et al., 2010). In this work, two kinds of basal tissue culture media were compared: DMEM and OM. OM has been widely used in the culture of bone marrow cells(Coelho and Fernandes, 2000), periosteal cells(Tate et al., 2004), primary calvarial osteoblasts(Orriss et al., 2007), and dura mater cells(Greenwald et al., 2000). DMEM promotes proliferation, whereas OM stimulates the differentiation of bone cells. However, there are no data regarding how long and how well mouse calvarial bone can survive in vitro. To examine bone resorption in organ culture, explants of bone have been maintained for short incubation times, usually 2-4 days(Meghji et al., 1998). Neonatal mouse calvarial bone is usually cultured for 7 days (Curtin et al., 2009; Paul et al., 2012), 10 days (Dibart et al., 2016a; Liu et al., 2013; Tong et al., 2016) or up to 2 weeks at most (Mohammad et al., 2008a). This may be because apoptosis of osteoclasts generally begins to occur after 4-12 days of in vitro culture (Loutit and Townsend, 1982; Marshall et al., 1987). The survival of mouse calvarial bone during the culture period warrants further investigation. Osteoclasts have been proven to be abundant in 4-day-old mouse calvarial bone and can be released mechanically by bone fragmentation (Hoebertz and Arnett, 2003). Osteoclast precursors are specifically activated to recognize bone surfaces and migrate to form multinucleated osteoclasts (Nakamura et al., 2012). Importantly, multinucleated osteoclasts do not form in dead bone tissue (Marshall and Rowlands, 2003). Tartrate-resistant acid phosphatase (TRAP)-positive osteoclast-like cells were therefore regarded as indicators of bone survival in vitro in this study.

When researchers evaluate the bone formation of cultured mouse calvariae, the traditional procedure involves bone quantitative histomorphometry, which not only is time consuming but also prevents the tested bones from being available for further testing. In contrast, non- invasive and high- 
resolution microcomputed tomography (micro-CT) has shown advantages in medical applications, especially with respect to bone research. However, an important consideration when utilizing micro$\mathrm{CT}$ is the effect of radiation on living bone. Radiation effects from micro-CT on living animals seem to depend on ionizing radiation doses (Mitchell and Logan, 1998; Tanvir et al., 2018), species, genetic strains (Klinck et al., 2008), age (Brouwers et al., 2007) and bone sites (Williams and Davies, 2006). There remains a lack of published data on the responses of cultured neonatal mouse calvarial bones to radiation doses from micro-CT. The objective of this study was to evaluate the bone formation of mouse calvariae in two different culture media by micro-CT technology and histomorphometry and to explore the effects of micro-CT radiation on cultured mouse calvarial bone.

\section{Materials and methods}

\subsection{Organ culture of neonatal mouse calvariae}

Heads (calvarial bones) of 4-day-old CD-1 female mice were provided by the Biological Support Facility of the University of Manchester and Chongqing Medical University. Mice were sacrificed by cervical dislocation and dissected under sterile conditions. During dissection, the dura mater and periosteum were kept intact on the bone surfaces. The mouse calvariae were exposed as shown in Fig. 5a. All experimental procedures were approved by the Ethics Committee of Chongqing Medical University, China (2013041).

The bones were cultured free-floating in 24-well tissue culture plates with DMEM (with glutamax, Invitrogen, Paisley, UK) supplemented with 10\% foetal bovine serum (Invitrogen, Paisley, UK), 100 $\mathrm{U} / \mathrm{ml}$ penicillin, and $100 \mathrm{mg} / \mathrm{ml}$ streptomycin (Invitrogen, Paisley, UK). One day later, the bones were randomly divided into 5 groups ( $\mathrm{n}=5 /$ group) for micro-CT (1072, SkyScan, BE) scanning and histological staining at weekly intervals according to the scanning and staining days (Table 1). The OM was composed of DMEM supplemented with foetal bovine serum, penicillin/streptomycin, dexamethasone (Dex) (Sigma-Aldrich,

Dorset, UK), ascorbic acid 2-phosphate solution (AA) (Sigma-Aldrich, Dorset, UK) and bglycerophosphate (bGP) (Sigma-Aldrich, Dorset, UK) as described in a previous article(Wu et al., 2014). The OM-control group was scanned only at the first and final time points. The bones were cultured in a humidified atmosphere of $5 \% \mathrm{CO} 2$ at $37{ }^{\circ} \mathrm{C}$. The culture media were changed every 3 days. 


\section{Table 1}

Groups tested by micro-CT scanning and histological staining

\begin{tabular}{|c|c|c|c|c|}
\hline $\begin{array}{l}\text { Group } \\
(\mathrm{n}=5)\end{array}$ & $\begin{array}{l}\text { Culture medium } \\
\text { on the } 1 \text { st day }\end{array}$ & $\begin{array}{l}\text { Culture medium after } \\
\text { the } 1^{\text {st }} \text { day }\end{array}$ & $\begin{array}{l}\text { Micro-CT scanning } \\
\text { days }\end{array}$ & $\begin{array}{l}\text { Histological staining } \\
\text { days }\end{array}$ \\
\hline DMEM & DMEM & DMEM & days $2,9,16$, and 23 & days $2,9,16$, and 23 \\
\hline $\mathrm{OM}$ & DMEM & $\mathrm{OM}$ & days $2,9,16$, and 23 & days $2,9,16$, and 23 \\
\hline OM- control & DMEM & $\mathrm{OM}$ & days 2 and 23 & \\
\hline
\end{tabular}

\subsection{Micro-CT analysis}

The cultured calvariae were scanned using micro-CT according to Table 1 . To obtain baseline measurements, the same medium (DMEM) was used on the first day for all groups. After the first day, different media were used. During scanning, the calvariae were enclosed in sterilized Eppendorf test tubes containing culture medium (Fig. 5b). There was a tightly fitting plastic tube inside each Eppendorf test tube that supported the calvaria. The Eppendorf test tube was fixed to a stub with plasticine. The bones were scanned with an X-ray tube potential of $100 \mathrm{kV}$ and a current intensity of $98 \mu \mathrm{A}$. Each calvaria was exposed for 3 seconds per projection. The rotation range was $180^{\circ}$, and an effective pixel size of $11 \mu \mathrm{m}$ was set. Images were obtained under $25 \times$ magnification. Care was taken to avoid contamination.

\subsection{Coefficient of variation}

The reproducibility of the measurements was evaluated in an adjoining study in which alcoholfixed calvarial bone was scanned three times on different days using the same scanning settings. Four bone structure parameters: the bone surface (BS), bone volume (BV), bone surface density (BS/TV(Tissue volume)) and percent bone volume (BV/ TV) were obtained. The coefficient of variation $(\mathrm{CV})$ was determined for $\mathrm{BV}$ and $\mathrm{BS}$ using the formula $\mathrm{CV}=$ standard deviation $(\mathrm{SD}) /$ mean.

\subsection{Histological and quantitative histomorphometric analyses}

The calvarial bones were fixed with 10\% neutral buffered formalin (Solarbio, Beijing, China) for $24 \mathrm{~h}$ and decalcified in 14\% ethylenediamine tetraacetic acid (EDTA; pH 7.2) at room temperature for $24 \mathrm{~h}$. The bones were subsequently dehydrated by exposure to an alcohol gradient and embedded. 
To guarantee that all calvarial bones were in the same orientation(Mohammad et al., 2008b), 5- $\mu$ mthick sections were collected along the intersection of the coronal suture and the posterior frontal suture. The cut direction for the histological sections was shown in Fig. 5a. Five different sections spaced at intervals of at least $100 \mu \mathrm{m}$ were selected from each specimen(Parfitt, 1988a). Some sections were stained with haematoxylin and eosin (H\&E) (Solarbio, Beijing, China) for histological observations(Dibart et al., 2016b). Other sections were processed for TRAP staining (Sigma-Aldrich, St. Louis, USA) according to the manufacturer's instructions. Dark red-stained multinucleated giant cells were considered TRAP-positive cells. Ten representative areas from each section were imaged using an Olympus BX41 microscope (Olympus, Tokyo, Japan) to which a camera (Olympus DP80, Japan) was attached. The images were captured with $20 \times$ objectives (UPlanFL N, 20×/0.50 $\infty / 0.17 /$ FN26.5, Olympus, Japan) using cellSens Standard software (Olympus, Japan). The images were analysed to assess the numbers of osteoblasts and osteoclasts using Image-Pro Plus software (Media Cybernetics L.P., Version: 6.0). The number of osteoblasts per unit BS (N. Ob/BS, $/ \mathrm{mm}^{2}$ ) and the number of osteoclasts per unit $\mathrm{BS}\left(\mathrm{N}\right.$. Oc/BS, $\left./ \mathrm{mm}^{2}\right)$ were determined by manually counting the osteoblasts and osteoclasts, respectively, and dividing the values by the bone area (Parfitt et al., 2010). Blinded analysis was used for the histological sections to prevent unintentional bias during microscopic analysis.

\subsection{Statistical analysis}

SPSS 22.0 software (Chicago, IL, USA) was used for statistical analysis. Graphs were performed using Graph Pad Prism 8 software (San Diego, California, USA). The data are presented as means \pm $\mathrm{SD}$. Independent-sample $\mathrm{t}$ tests were used to analyse the differences between the bones cultured in DMEM and those cultured in OM at each single culture time point. Furthermore, two-way analysis of variance (ANOVA) with repeated measures was used to test the first and last measurements of the calvariae on days 2 and 23. For all statistical tests, $p<0.05$ was considered to indicate statistical significance.

\section{Results}

3.1. Histological observations of calvarial bone

Histological observations that illustrate the effect of either DMEM or OM on calvariae are presented in Fig. 1. Images of H\&E-stained histological sections are shown in Fig. 1a-h. There was almost no observable new osteoid/bone formation in either group on day 2 (Fig. 1a-b). The newly formed calvarial bone increased in size and width progressively from days 9 to 23 . The darkercoloured areas represent old bone, whereas the lighter areas represent new bone (Fig. 1c-h). This 
newly formed bone was mostly restricted to the inner surface of the periosteum. Active osteoblasts were observed during the formation of new bone, and sites of new bone were counted to measure new bone formation (Garrett, 2003b). Images of TRAP-stained histological sections are shown in Fig. 1i-p. Irregularly shaped TRAP-positive osteoclasts, which were mainly distributed on the endocranial membrane, were visible until day 16. TRAP staining of sections was performed to determine whether the bone tissues survived in vitro.

\subsection{Histomorphometric measurements}

The results of quantitative histomorphometric analysis of H\&E sections and TRAP-stained sections from both groups are shown in Fig. 2. The N. Ob/BS $\left(/ \mathrm{mm}^{2}\right)$ for the DMEM group was significantly smaller than that for the OM group on days 9 ( $p<0.01), 16(p<0.01)$, and 23 ( $p<$ 0.05 ) (Fig. 2a). The opposite trend was observed for N. Oc/BS $\left(/ \mathrm{mm}^{2}\right)$ on days $9(p<0.05)$ and $16(p$ $<0.05$ ) (Fig. 2b). Almost no osteoclasts were observed on day 23 in either group. The statistical analysis indicated that culture of calvariae in OM increased the number of osteoblasts but decreased the number of osteoclasts.

\subsection{Micro-CT assessment of bone formation}

Three-dimensional (3D) reconstructions of the mouse calvariae were created based on twodimensional (2D) cross-sections. The 3D images could easily be rotated in the $\mathrm{x}-, \mathrm{y}-$, and z-planes. Fig. 3 shows representative images of the calvariae on days 2, 9, 16, and 23. Bone formation can be observed in the images. The results of quantitative analyses of the bone structure parameters are presented in Fig. 4. The BS, BV, BS/TV and BV/TV of calvariae cultured in both types of media increased during the culture period. There were statistically significant differences in BS, BV, $\mathrm{BS} / \mathrm{TV}$ and BV/TV $(p<0.05)$ between the DMEM- differences of bone parameters were observed between the OM group calvariae and the OM-control group calvariae on days 2 and $23(p>0.05)$. The CV was less than $1.4 \%$ for all bone parameters.

\section{Discussion}

In mouse calvarial organ cultures, the periosteum and dura mater play important roles in the process of bone formation. The periosteum is a connective tissue anchored to bone, and it has two layers: an outer fibrous layer containing fibroblasts, vessels and fibres of Sharpey and an inner cambium layer containing nerves, capillaries, osteoblasts and undifferentiated mesenchymal stem cells (Malizos and Papatheodorou, 2005). The inner layer serves as a reservoir of undifferentiated mesenchymal cells 
capable of differentiation into osteoblastic lineages and as a source of growth factors that are important in the bone formation process ( $\mathrm{Li}$ et al., 2004). The dura mater is the external membrane covering the brain. It consists of a periosteal layer and a meningeal layer. The ossification capacity of immature animals has been well documented. This capacity may be related to osteoinductive or osteoconductive qualities of the immature dura and seems to be lost with advancing age (Greenwald et al., 2000).

Calvarial bone formation ability depends on the culture medium, which influences cell proliferation and differentiation. In this work, DMEM and OM were compared. OM contains bGP, Dex and AA, all of which are known to promote the osteoblastic phenotype. Dex induces osteoblast differentiation by reducing cell growth and increasing alkaline phosphatase (ALP) activity and bonelike formation in cell cultures (Beloti and Rosa, 2005; Yamanouchi et al., 1997). Dex also increases cell growth when used either alone or in combination with AA and bGP (Coelho and Fernandes, 2000). AA is a stable ascorbic acid derivative. AA stimulates the synthesis and maturation of collagen molecules and increases the ALP activity of osteoblastic cell cultures (Hata and Senoo, 1989; Owen et al., 1990). Cultured cells showed more osteoblast differentiation and less cell proliferation when bGP is present than when it is absent. Without bGP, bone cells fail to form calcium phosphate deposits after up to 42 days of culture in $\alpha$-modified minimal essential medium $(\alpha$-MEM). Although no mineralization was found in cell cultures that lacked bGP, our data and 3D images showed that the calvariae cultured without bGP had continuous bone formation over 23 days. Bone organ culture is different from cell culture in that mineralization is already present in calvariae. It has been reported that mineralization, once started, will proceed without exogenous organic or inorganic phosphate (Bellows et al., 1991). Similar concentrations of inorganic phosphate (sodium phosphate monobasic) are present in $\alpha$-MEM (122 mg/L) and DMEM (125 mg/L). Because of the osteogenic supplements in $\mathrm{OM}$, and especially because of the greater amount of exogenous phosphate in OM, the OM resulted in much more bone formation than the DMEM during the culture period in this study. The calvariae could be maintained in the culture media for at least 23 days, during which bone formation indexes increased. Nevertheless, the greatest increases were observed during the first week.

Most calvarial bone surfaces undergo either resorption or formation, and virtually all cells on the surface are either osteoclasts or osteoblasts in young, rapidly growing small animals (Parfitt, 1988b). Active osteocytes, especially multinucleated osteoclasts, are distributed on the bone and endocranial membrane. The numbers of TRAP-positive osteoclasts and/or the areas of resorption pits were historically assessed to determine bone resorption until micro-CT became the widespread standard for measuring resorption. Fig. 2 illustrates the effects of both DMEM and OM on bone formation of 
neonatal mouse calvariae; the results were as expected. The histomorphometric measurements showed that the OM group had a significantly greater N. Ob/BS than the DMEM group. However, quantitative histomorphometry measurements cannot provide precise data because of the complexity of the procedure and the inevitability of unintended human error. In addition, the measurements are performed on 2D sections, which are susceptible to the nonuniformity of biological specimens.

In this study, the number of TRAP-positive osteoclasts was regarded as an important reference index for the survival of bone in vitro. TRAP- positive osteoclasts resided almost exclusively on the endocranial membrane and were found to survive after an incubation period of 16 days. However, simple cultured osteoclasts lived for only 4-12 days in vitro. Tatsuo et al. similarly reported that isolated mouse osteoclasts have a limited lifespan of less than 1 week (Nakamura et al., 2012). It has been hypothesized that the presence of a bone matrix environment around bone cells has an appreciable impact on the survival time of osteoclasts. Osteoblasts can communicate with osteoclasts through diffusible paracrine factors, cell-cell contact and cell-bone matrix interaction (Matsuo and Irie, 2008). In addition, osteoblasts and bone marrow stromal cells have been shown to regulate osteoclasts via RANK/RANKL (Chambers, 2000; Takahashi et al., 1988) and M-CSF/c-Fms interactions (Elsegood et al., 2006). Importantly, organ culture preserves not only the interrelationships among the different cell types in bone but also the relationships between cells and the bone matrix. Accumulating evidence has collectively indicated that osteoclasts maintained in vitro organ culture systems have longer life spans than those maintained in traditional cell culture systems. Notably, this study was intended not to prove that bone organ culture is superior to cell culture systems or animal models but to provide a way to study bone metabolism.

Several biological markers are commonly used to assess osteoblast differentiation, such as ALP and osteocalcin. The final stage of differentiation is mineral deposition in the extracellular matrix. Micro- CT distinguishes bone from other materials based on the extent of mineralization. Immature bone established from a matrix composed of proteins may not absorb or deflect radiation in the same way that mature bone does (Recinos et al., 2004). Micro-CT provides a way to evaluate the bone formation of cultured mouse calvariae. Although micro-CT cannot replace histology, this noninvasive technique does not require sample preparation in the way that the traditional light microscope method does, and micro-CT has good reproducibility. With micro-CT technology, it is possible to measure the bone structure and generate $3 \mathrm{D}$ images of the same sample over time. However, repeated exposure of the same sample to radiation in longitudinal studies may cause damage to cells. Damage to osteogenic cells could affect bone formation and thereby alter bone structure (Brouwers et al., 2007). Klinck (Klinck et al., 2008) evaluated the effects of micro-CT radiation on bone architecture in mice and rats in vivo. The effects of radiation on mice were 
dependent on the mouse strains. Similar findings have been reported for rats (Brouwers et al., 2007). These results suggest that the effects of radiation are related to the radiation dose, the species and the study design. A dose of $400 \mathrm{mGy}$ or less has been found to have no effects on the proliferation and differentiation of osteoblast-like cells in vitro (Dare et al., 1997). However, a high dose (> 2 Gy) has been found to reduce proliferation rates and collagen production in osteoblasts (Gal et al., 2000). Matsumura (Matsumura et al., 1996) reported that high-dose irradiation induces osteoblast differentiation and calcification. However, differentiated osteoblasts are unlikely to proliferate, which increases the likelihood of diminished bone growth. In this study, continuous bone formation was found in the calvariae cultured in both types of media. For determination of the radiation effect, a baseline scan was unavoidable. If the baseline radiation had any effects on the morphological parameters of the cultured bones, weekly repeated scans would have had much greater effects on them. However, no differences of bone indexes were found in the calvariae scanned 4 times, which suggests that radiation did not significantly affect the cultured mouse calvariae under this study design.

\section{Conclusions}

Micro-CT has certain advantage in displaying three-dimensional bone parameters. Meanwhile, histological technology has advantage in displaying reflecting local cell morphology. Within the limitations of this study, the results implied that bone formation of cultured neonatal mouse calvaria continued for at least 16 days in both media, suggesting that mouse calvaria could survive well in vitro during this period. Bone formation ability was higher in OM cultures.

\section{Authors' contributions}

All authors contributed to the study conception and design. The material preparation, data collection and data analysis were performed by Ren-jian Zheng, Jin-lin Song, and David C. Watts. The first draft of the manuscript was written by Ren-jian Zheng and Xiao-hong Wu. All authors commented on previous versions of the manuscript. All authors read and approved the final manuscript.

\section{Funding}

This study was funded by the National Natural Science Foundation of China (grant number 81970914); the Chongqing Science \& Technology Commission, China (grant number cstc2017jcyjAX0215); the Chongqing Yuzhong Science and Technology Project, China (grant number 20170117); and a project supported by the program for innovation team building at institutions of higher education in Chongqing in 2016. 


\section{Ethics approval}

All experimental procedures were approved by the Ethics Committee of Chongqing Medical University (2013041).

Consent to participate Not applicable.

Consent for publication Not applicable.

\section{Declaration of Competing Interest}

The authors report no declarations of interest.

\section{Acknowledgements}

The authors thank the Stomatological Hospital of Chongqing Medical University for its infrastructure and funding support.

\section{References}

Bellows, C.G., Aubin, J.E., Heersche, J.N.M., 1991. Initiation and progression of mineralization of bone nodules formed in vitro: the role of alkaline phosphatase and organic phosphate. Bone \& Mineral. 14, 27-40.

Beloti, M.M., Rosa, A.L., 2005. Osteoblast differentiation of human BM cells under continuous and discontinuous treatment with dexamethasone. Brazilian Dental Journal. 16, 156-161.

Brouwers, J.E.M., Van Rietbergen, B., Huiskes, R., 2007. No effects of in vivo micro-CT radiation on structural parameters and bone marrow cells in proximal tibia of wistar rats detected after eight weekly scans. Journal of Orthopaedic Research. 25, $1325-1332$.

Chambers, T.J., 2000. Regulation of the differentiation and function of osteoclasts. Journal of Pathology. 192, 4-13.

Cochran, D.L., Rouse, C.A., 1993. The effect of conditioned medium from connective tissue fibroblasts and epithelium on calcium release from mouse calvarial organ culture. Archives of Oral Biology 38, 61.

Coelho, M.J., Fernandes, M.H., 2000. Human bone cell cultures in biocompatibility testing. Part II: effect of ascorbic acid, beta-glycerophosphate and dexamethasone on osteoblastic differentiation. Biomaterials 21, 1095-1102.

Curtin, P., McHugh, K.P., Zhou, H.-Y., Fluckiger, R., Goldhaber, P., Oppenheim, F.G., Salih, E., 2009. Modulation of Bone Resorption by Phosphorylation State of Bone Sialoprotein. Biochemistry 48, 6876-6886.

Dare, A., Hachisu, R., Yamaguchi, A., Yokose, S., Yoshiki, S., Okano, T., 1997. Effects of Ionizing Radiation on Proliferation and Differentiation of Osteoblast-like Cells. Journal of Dental Research. 76, 658-664. https://doi.org/10.1177/ 00220345970760020601.

Dibart, S., Alasmari, A., Zanni, O., Salih, E., 2016a. Effect of Corticotomies with Different Instruments on Cranial Bone Biology Using an Ex Vivo Calvarial Bone Organ Culture Model System. International Journal of Periodontics \& Restorative Dentistry. 36, s123-s136. 
Dibart, S., Alasmari, A., Zanni, O., Salih, E., 2016b. Effect of Corticotomies with Different Instruments on Cranial Bone Biology Using an Ex Vivo Calvarial Bone Organ Culture Model System. International Journal of Periodontics \& Restorative Dentistry 36 (Suppl), s123.

Elsegood, C.L., Zhuo, Y., Wesolowski, G.A., Hamilton, J.A., Rodan, G.A., Duong, L.T., 2006. M$\mathrm{CSF}$ induces the stable interaction of cFms with $\alpha \mathrm{V} \beta 3$ integrin in osteoclasts. The International Journal of Biochemistry \& Cell Biology 38, 0-1529.

Gal, T.J., Munoz-Antonia, T., Muro-Cacho, C.A., Klotch, D.W., 2000. Radiation Effects on Osteoblasts In Vitro: A Potential Role in Osteoradionecrosis. Arch Otolaryngol Head Neck Surg. 126, 1124-1128. https://doi.org/10.1001/archotol.126.9.1124.

Garrett, I.R., 2003a. Assessing bone formation using mouse calvarial organ cultures. Methods Mol Med 80, 183.

Garrett, I.R., 2003b. Assessing bone formation using mouse calvarial organ cultures. Methods Mol Med. 80, 183-198. https://doi.org/10.1385/1-59259-366-6:183.

Goldhaber, P., Rabadjija, L., 1991. Calcium 'homeostatic' effect of 1 alpha,25dihydroxycholecalciferol on young adult mouse calvaria in organ culture.

Mechanisms of Ageing and Development 60, 25-34.

Greenwald, J.A., Mehrara, B.J., Spector, J.A., Fagenholz, P.J., Saadeh, P.B., Steinbrech, D.S., Gittes, G.K., Longaker, M.T., 2000. Immature versus mature dura mater: II. Differential expression of genes important to calvarial reossification.

Plastic \& Reconstructive Surgery. 106, 630.

Hata, R., Senoo, H., 1989. L-ascorbic acid 2-phosphate stimulates collagen accumulation, cell proliferation, and formation of a three-dimensional tissuelike substance by skin fibroblasts. Journal of Cellular Physiology 138, 8-16.

Hoebertz, A., Arnett, T.R., 2003. Isolated Osteoclast Cultures.

Klinck, R.J., Campbell, G.M., Boyd, S.K., 2008. Radiation effects on bone architecture in mice and rats resulting from in vivo micro-computed tomography scanning. Medical Engineering \& Physics. 30, 888-895.

Li, M., Amizuka, N., Oda, K., Tokunaga, K., Ito, T., Takeuchi, K., Takagi, R., Maeda, T., 2004. Histochemical evidence of the initial chondrogenesis and osteogenesis in the periosteum of a rib fractured model: Implications of osteocyte involvement in periosteal chondrogenesis. Microscopy Research and Technique. 64, 330-342.

Liu, J., Czernick, D., Lin, S.-C., Alasmari, A., Serge, D., Salih, E., 2013. Novel bioactivity of phosvitin in connective tissue and bone organogenesis revealed by live calvarial bone organ culture models. Developmental Biology. 381, 256-275.

Loutit, J.F., Townsend, K.M.S., 1982. Longevity of osteoclasts in radiation chimeras of

osteopetrotic beige and normal mice. British Journal of Experimental Pathology. 63, 221-223.

Malizos, K.N., Papatheodorou, L.K., 2005. The healing potential of the periosteum: Molecular aspects. Injury-international Journal of the Care of the Injured. 36, S13-S19.

Marshall, M.J., Rees, J.A., Nisbet, N.W., Wiseman, J., 1987. Reduced life span of the osteoclast in osteopetrotic (mi and midi) mice. Bone \& Mineral. 2, 115-124.

Marshall, M.J., Rowlands, M.N., 2003. Analysis of Osteoclast Function in Mouse Calvarial Cultures. Methods in Molecular Medicine 80, 169-182.

Matsumura, S., Jikko, A., Hiranuma, H., Deguchi, A., Fuchihata, H., 1996. Effect of X-Ray Irradiation on Proliferation and Differentiation of Osteoblast. Calcified Tissue International. 59, 307-308.

Matsuo, K., Irie, N., 2008. Osteoclast-osteoblast communication. Arch Biochem Biophys. 
473, 201-209. https://doi.org/10.1016/j.abb.2008.03.027.

Meghji, S., Hill, P.A., Harris, M., 1998. Bone organ cultures.

Mitchell, M.J., Logan, P.M., 1998. Radiation-induced changes in bone. In: Radiographics A Review

Publication of the Radiological Society of North America Inc., 18, pp. 1125-1136.

Mohammad, K.S., Chirgwin, J.M., Guise, T.A., 2008a. Assessing New Bone Formation in Neonatal Calvarial Organ Cultures.

Mohammad, K.S., Chirgwin, J.M., Guise, T.A., 2008b. Assessing new bone formation in neonatal calvarial organ cultures. Methods Mol Biol. 455, 37-50. https://doi.org/ 10.1007/978-1-59745104-8_3.

Nakamura, I., Takahashi, N., Jimi, E., Udagawa, N., Suda, T., 2012. Regulation of osteoclast function. Journal of Bone \& Mineral Research the Official Journal of the American Society for Bone \& Mineral Research. 22, 11.

Orriss, I.R., Utting, J.C., Andrea, B.B., Kay, C., Grubb, B.R., Geoffrey, B., Arnett, T.R., 2007.

Extracellular nucleotides block bone mineralization in vitro: evidence for dual

inhibitory mechanisms involving both $\mathrm{P} 2 \mathrm{Y} 2$ receptors and pyrophosphate.

Endocrinology. 148, 4208-4216.

Owen, T.A., Aronow, M., Shalhoub, V., Barone, L.M., Wilming, L., Tassinari, M.S., Kennedy, M.B., Pockwinse, S., Lian, J.B., Stein, G.S., 1990. Progressive development of the rat osteoblast phenotype in vitro: reciprocal relationships in expression of genes associated with osteoblast proliferation and differentiation during formation of the bone extracellular matrix. Journal of Cellular Physiology 143, 420-430.

Parfitt, A.M., 1988a. Bone histomorphometry: Proposed system for standardization of nomenclature, symbols, and units. Calcified Tissue International. 42, 284-286.

Parfitt, A.M., 1988b. Bone histomorphometry: proposed system for standardization of nomenclature, symbols, and units. Calcif Tissue Int. 42, 284-286.

Parfitt, A.M., Drezner, M.K., Glorieux, F.H., Kanis, J.A., Malluche, H., Meunier, P.J., Ott, S.M., Recker, R.R., 2010. Bone histomorphometry: standardization of nomenclature, symbols, and units. Report of the ASBMR Histomorphometry Nomenclature Committee. Journal of Bone \& Mineral Research. 2, 595-610.

Paul, Curtin, Helen, Youm, Erdjan, Salih, 2012. Three-dimensional cancer-bone metastasis model using ex-vivo co-cultures of live calvarial bones and cancer cells.

Biomaterials 33, 1065-1078.

Recinos, R.F., Hanger, C.C., Schaefer, R.B., Dawson, C.A., Gosain, A.K., 2004. Microfocal CT: a method for evaluating murine cranial sutures in situ. Journal of Surgical Research 116, 322-329.

Sakai, R., Eto, Y., Hirafuji, M., Shinoda, H., 2000. Activin release from bone coupled to bone resorption in organ culture of neonatal mouse calvaria. Bone 26, 235-240. Takahashi, N., Akatsu, T., Udagawa, N., Sasaki, T., Yamaguchi, A., Moseley, J.M., Martin, T.J., Suda, T., 1988.

OSTEOBLASTIC CELLS ARE INVOLVED IN OSTEOCLAST FORMATION. Endocrinology 123, 2600-2602.

Tanvir, Mustafy, Aur' elie, Benoit, Irene, Londono, Florina, Moldovan, Isabelle, Villemure,' 2018. Can repeated in vivo micro-CT irradiation during adolescence alter bone microstructure, histomorphometry and longitudinal growth in a rodent model? PLoS One. 15, e0207323.

Tate, M.L.K., Adamson, J.R., Tami, A.E., Bauer, T.W., 2004. The osteocyte. International Journal of Biochemistry \& Cell Biology. 36, 1-8.

Tong, X., Lu, W., You, T., Yan, J., Feng, D., Xiao-Hong, W., 2016. The Function of Naringin in Inducing Secretion of Osteoprotegerin and Inhibiting Formation of 
Osteoclasts. Evidence-Based Complementary and Alternative Medicine. 1-7, 2016.

Williams, H.J., Davies, A.M., 2006. The effect of X-rays on bone: a pictorial review. European Radiology 16, 619-633.

Wu, X., Downes, S., Watts, D.C., 2010. Evaluation of critical size defects of mouse calvarial bone: An organ culture study. Microsc Res Tech. 73, 540-547.

Wu, X., Wang, L., Deng, F., Watts, D.C., 2014. Mouse calvarial defect Model: An approach for the micro-tomographic evaluation of polymer scaffolds. Microscopy Research \& Technique. 77, 1037-1043.

Yamanouchi, K., Gotoh, Y., Nagayama, M., 1997. Dexamethasone enhances differentiation of human osteoblastic cells in vitro. Journal of Bone \& Mineral Metabolism. 15, 23-29. 

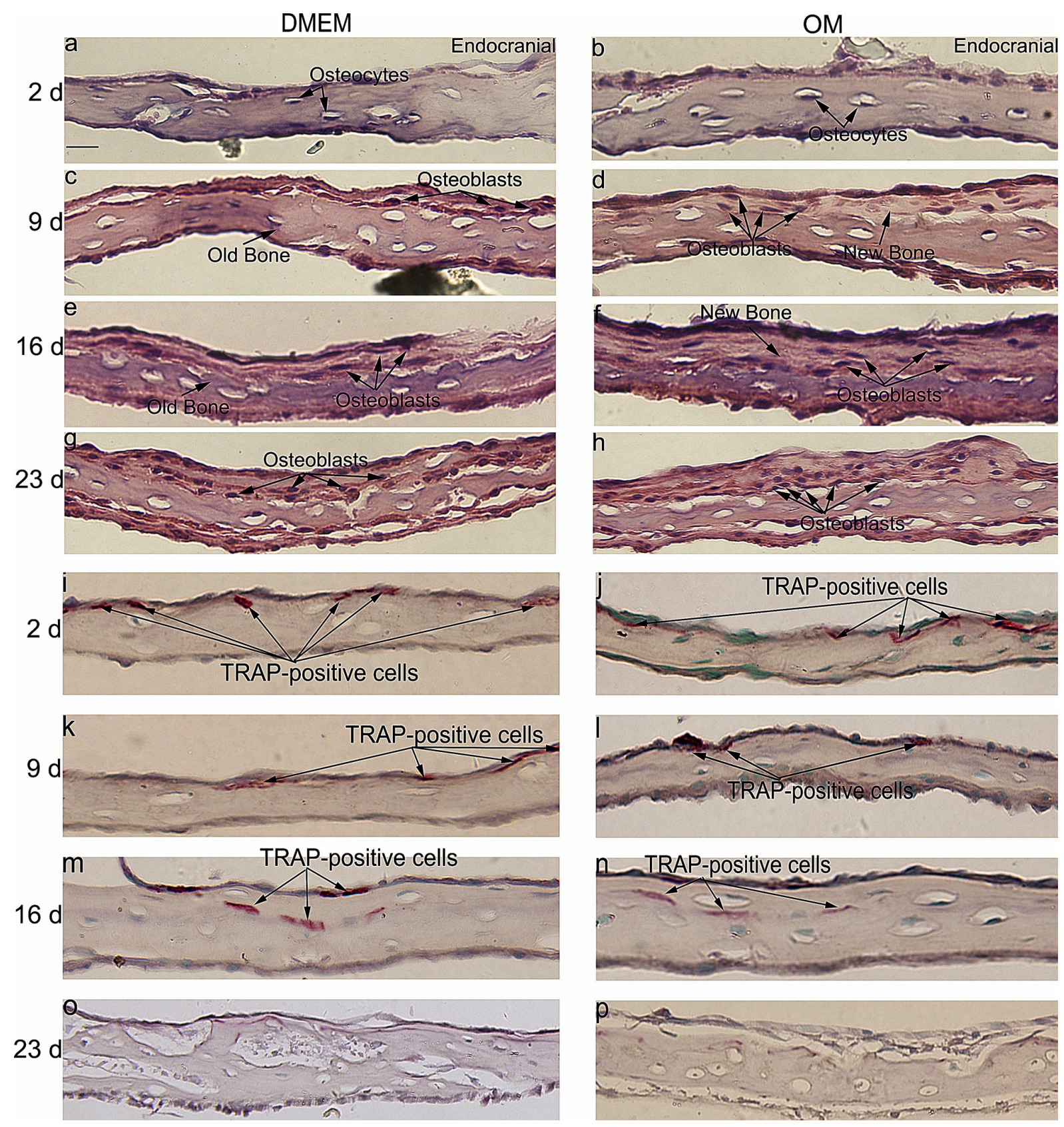

Fig. 1. H\&E/TRAP staining of sections of neonatal calvarial bone in the DMEM and OM groups. H\&E-stained sections of neonatal calvarial bone from the DMEM and OM groups on days 2 to 23 are shown (a-h). No obvious new osteoid/bone formation was observed on day 2 in either group (a, b). Representative sections from both groups showing new osteoid/bone and active osteoblast formation (c-h). TRAP-stained sections of neonatal calvarial bone from the DMEM and OM groups on days 2 to 23 are shown (i-p). No obvious TRAP-positive cells were observed on day 23 in either group $(\mathbf{o}, \mathbf{p})$. All magnifications are $200 \times$. The scale bar indicates $50 \mu \mathrm{m} . \mathrm{n}=5 /$ group. 

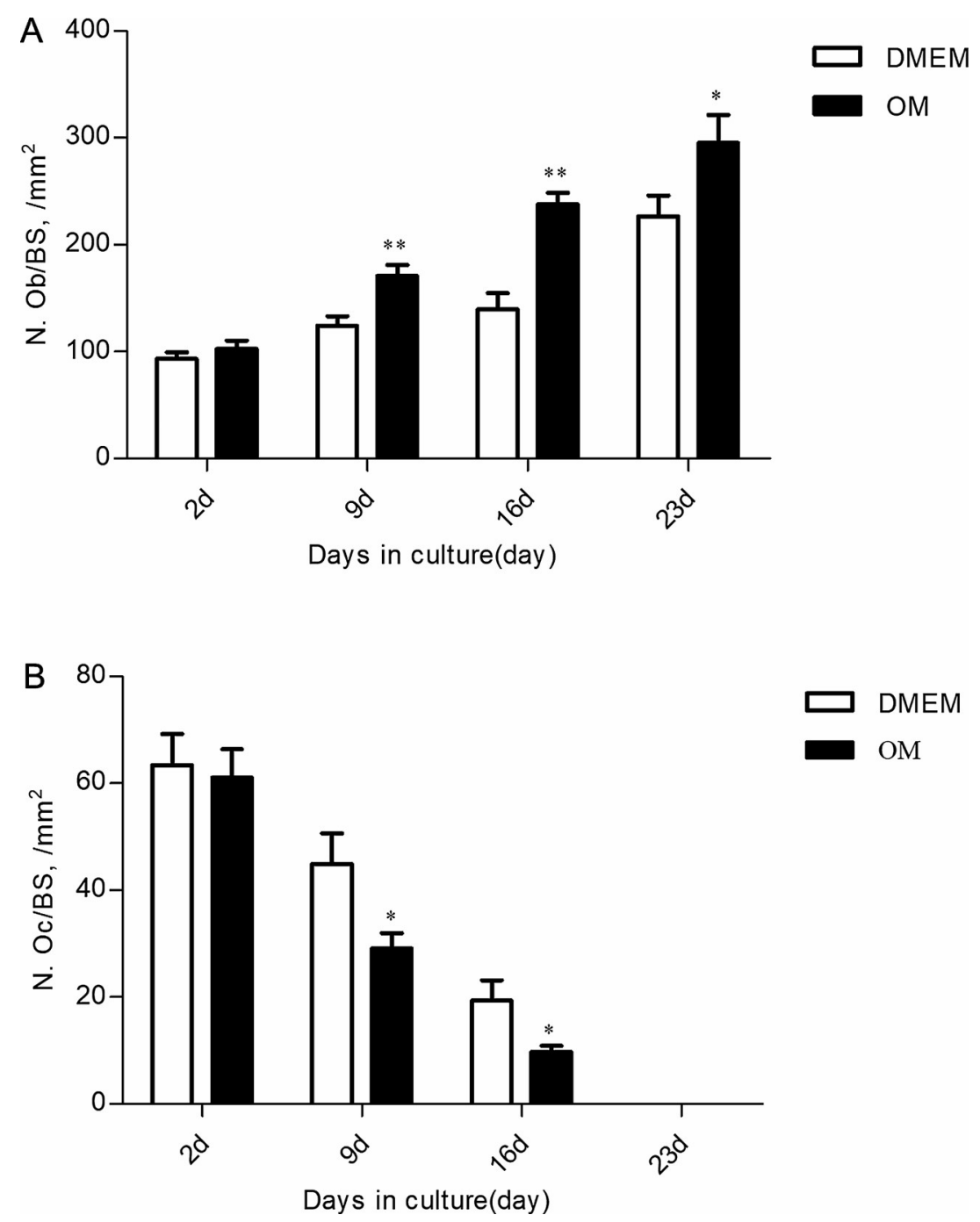

Fig. 2. Histomorphometric measurements of N. Ob/BS and N. Oc/BS in the DMEM and OM groups from days 2 to 23. The N. Ob/BS in the OM group was greater than that in the DMEM group on days $9(P<0.01), 16(P<0.01)$, and $23(P<0.01)(a)$. The N. Oc/BS in the OM group was lower than that in the DMEM group on days $9(P<0.05)$ and $16(P<0.05)(\mathbf{b})$. The lines above the bars indicate the SDs. ${ }^{*} P<0.05$ and $* * P<0.01$ versus DMEM group. $\mathrm{n}=5$ /group. 

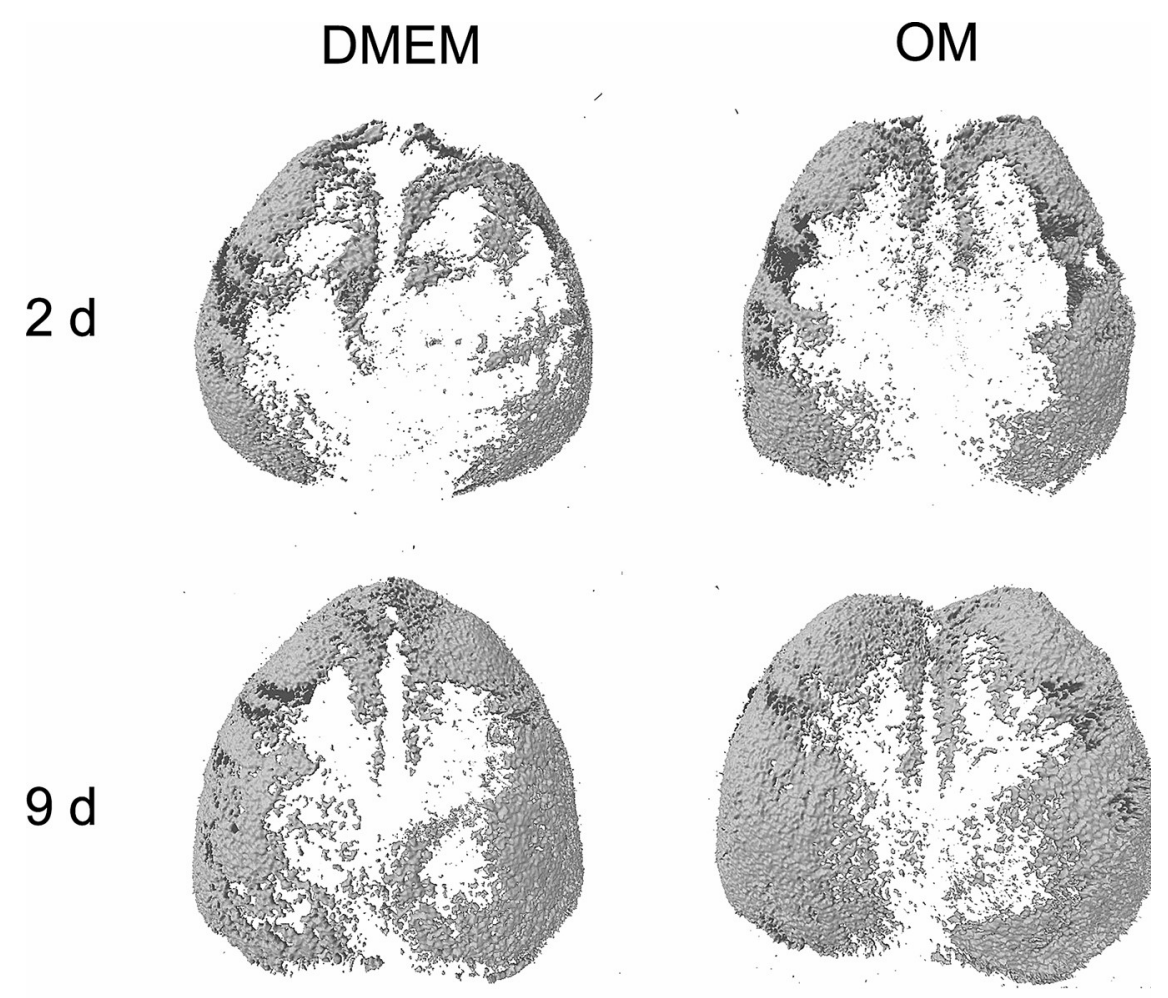

$16 \mathrm{~d}$
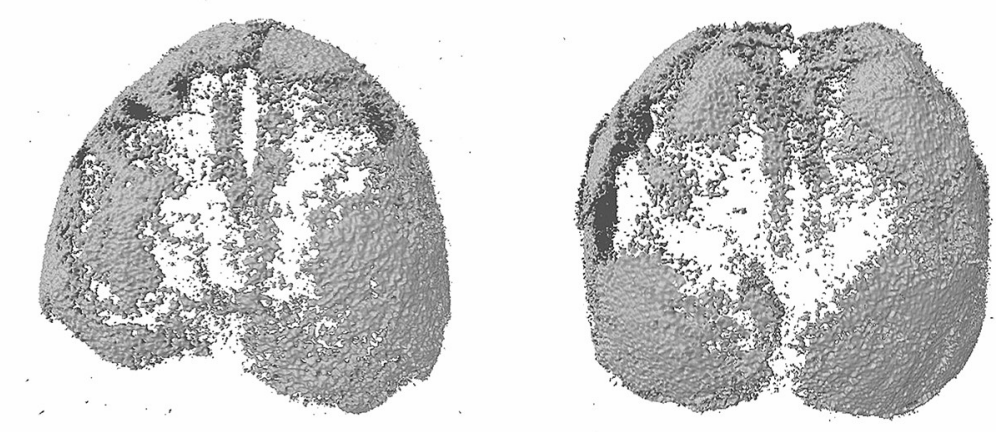

$23 d$
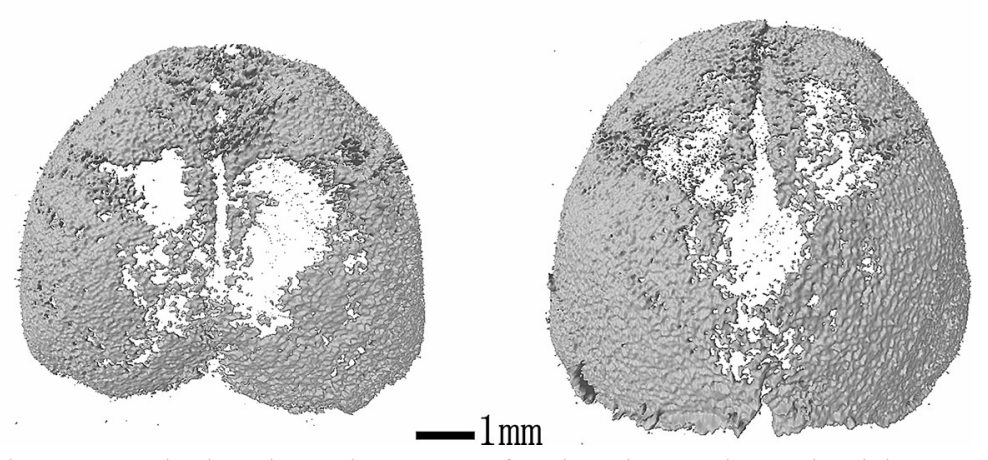

Fig. 3. Micro-CT-derived 3D images of calvariae cultured with DMEM and OM on days 2, 9, 16 and 23. Bone formation was observed from days 2 to 23. At each single culture time point, more bone was observed in the OM-cultured calvariae than in the DMEM-cultured calvariae, except at baseline. $n=5 /$ group. 

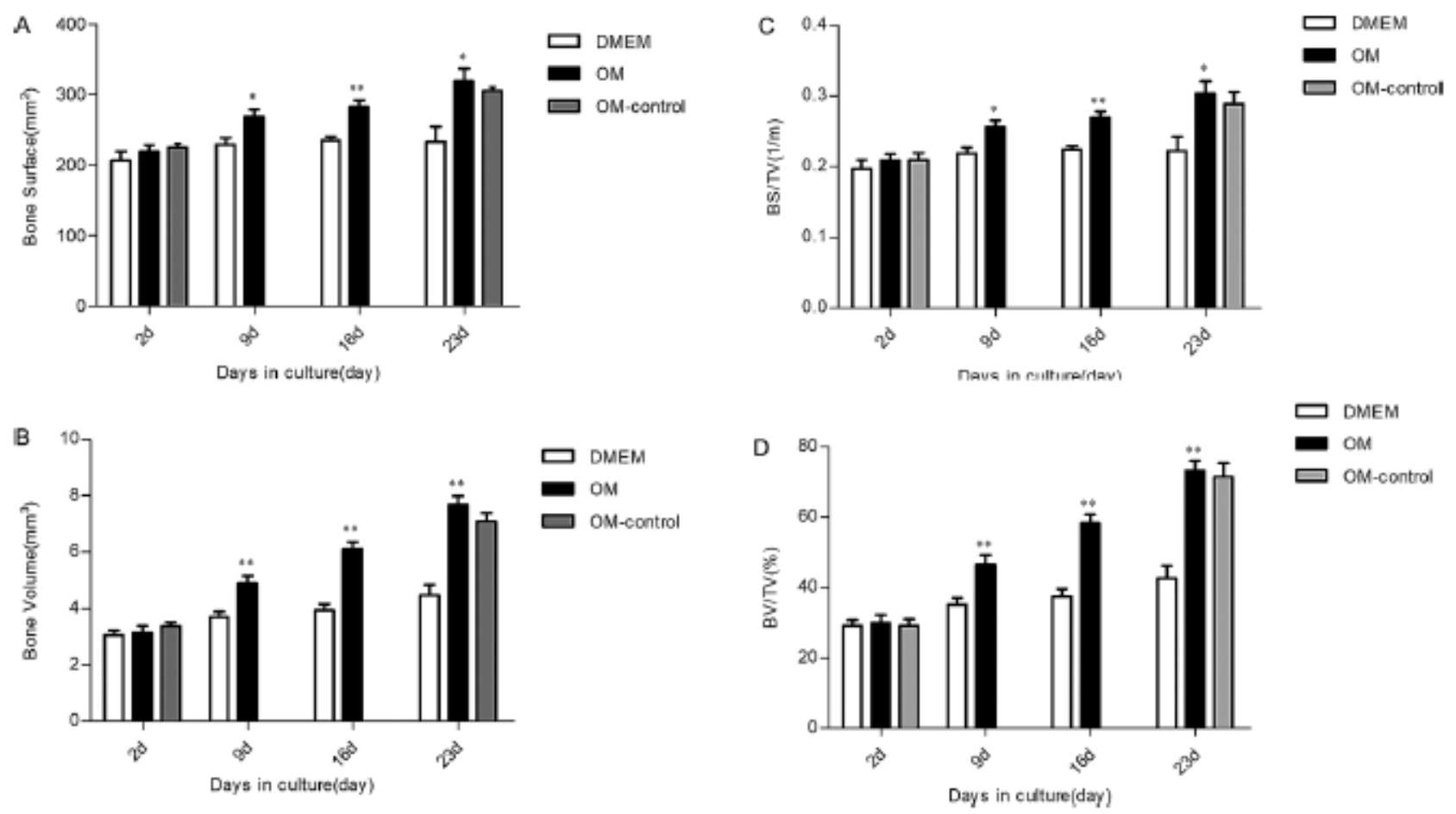

Fig. 4. BS (a), BV (b), BS/TV(c) and BV/TV(d) of calvaria cultured in two kinds of media on days 2, 9,16 and 23 . The lines above the bars indicate the SDs. $* P<0.05$ and $* * P<0.01$ versus DMEM group. $n=5 /$ group. 


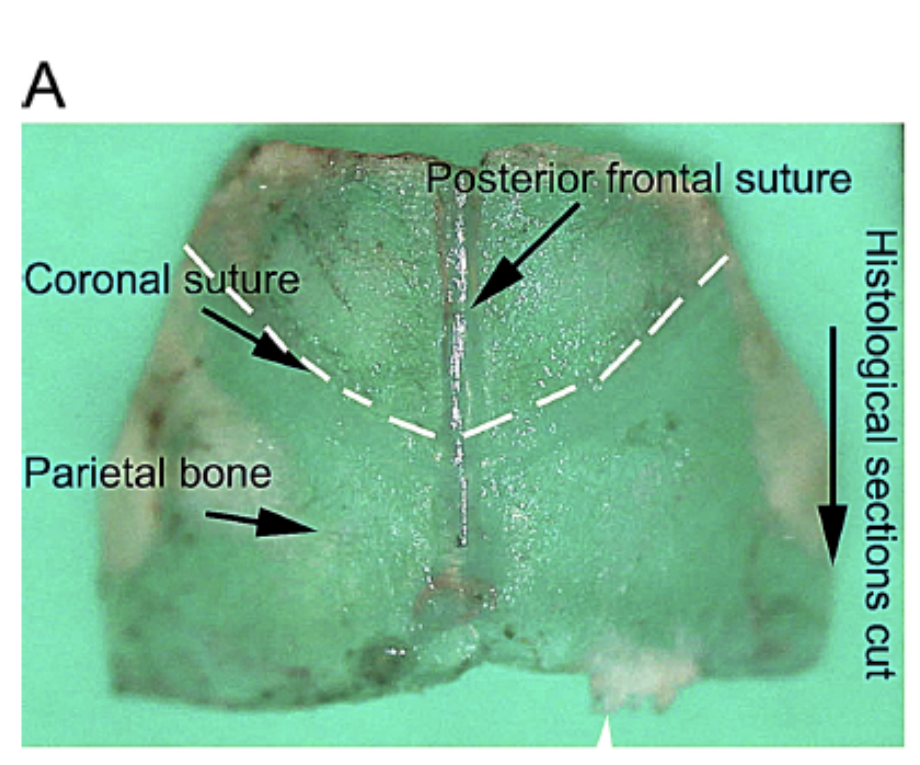

Calvarial bone

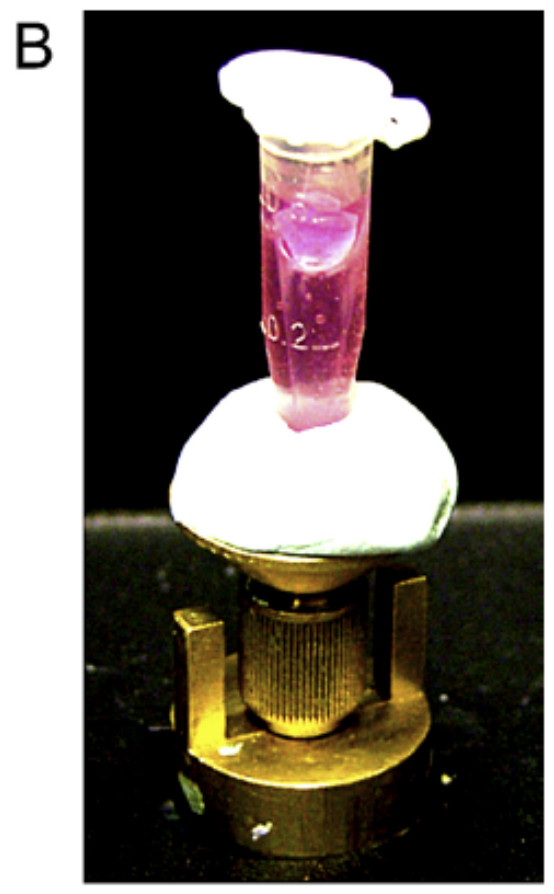

Sample holder

Fig. 5. Dorsal aspect of 4-day-old mouse calvarial bone and the main anatomical landmarks (a). The device used to hold the calvarial bone samples during scanning 